\title{
Field ionization detection of helium using a planar array of carbon nanotubes
}

\author{
K. M. O’Donnell, ${ }^{1,2}$ A. Fahy, ${ }^{1}$ M. Barr, ${ }^{1}$ W. Allison, ${ }^{3}$ and P. C. Dastoor ${ }^{1, *}$ \\ ${ }^{1}$ Centre for Organic Electronics, University of Newcastle, Callaghan, NSW 2308, Australia \\ ${ }^{2}$ The Bristol Centre for Nanoscience and Quantum Information, University of Bristol, Tyndall Avenue, Bristol BS8 1FD, United Kingdom \\ ${ }^{3}$ Cavendish Laboratory, University of Cambridge, Madingley Road, Cambridge, CB3 OHE, United Kingdom
}

(Received 21 June 2011; revised manuscript received 12 February 2012; published 15 March 2012)

\begin{abstract}
In this work we demonstrate the field ionization of neutral helium using a carbon nanotube forest in a parallelplate detector geometry. The nanotube forest was grown using plasma-enhanced chemical vapor deposition on a silicon substrate. With a high-positive voltage applied to the nanotubes, the measured ion current was directly correlated with the helium partial pressure. Moreover, we show that multiple nanotubes act as field ionization sources suggesting that, with careful nanotube engineering, significantly larger numbers of nanotubes should be able to contribute to the measured current, thus paving the way for high-efficiency, spatially resolved field ionization detection.
\end{abstract}

DOI: 10.1103/PhysRevB.85.113404

PACS number(s): 79.70.+q, 85.35.Kt, 07.77.-n

Introduction. There is a need for a compact, high-efficiency and high-sensitivity detector for neutral helium. Supersonic helium beams with a narrow velocity distribution can be produced with intensity and brightness similar to thermionic electron sources, ${ }^{1-3}$ while simultaneously possessing only thermal energies in the range $10-100 \mathrm{meV}$. Such a beam is therefore the ideal surface probe, and the development of a microscope based on a focused neutral helium beam has been the desired goal of much research in the past two decades. ${ }^{4-6}$ However, as helium has the highest ionization energy of all elements and a small electron impact cross section, ${ }^{7}$ it is difficult to detect efficiently. Field ionization (FI) offers near-unity detection efficiency in a very small spatial region without increasing the detector time constant.

Field ionization is the basis of the field ion microscope (FIM) and, for helium, requires fields of order $10^{10} \mathrm{~V} / \mathrm{m} .{ }^{8,9}$ Such fields can be generated by the application of a high voltage to the tips of sharp needles with radii of order 10-100 nm, traditionally fabricated from refractory metals such as tungsten. Field-induced evaporation of the tip material imposes an upper limit $\left(5.7 \times 10^{10} \mathrm{~V} / \mathrm{m}\right.$ for tungsten $\left.{ }^{8}\right)$ on the fields that can be applied over an extended period. Since even the refractory metals field evaporate under the conditions required for high-efficiency ionization of helium, field ionization detection of helium has not been regarded as practical. Furthermore, the sharp tips are by necessity of a very small radius, leading to an ionization area of the order tens to hundreds of square nanometers. The helium sensitivity of such detectors is therefore limited despite their high efficiency. Low sensitivity and low count rates drastically limit the speed with which a scanning helium microscope could operate, resulting in long acquisition times. ${ }^{6}$

Vertically aligned carbon nanotubes (CNTs) are inherently of the size scale required for field ionization, can be grown in massively parallel arrays consisting of millions of aligned tips, ${ }^{10}$ and should show strong resilience to high fields due to the strength of the $s p^{2}$ bonding within the walls of the tubes. ${ }^{11}$ Indeed, high-field vacuum microelectronic applications for carbon nanotubes have been pursued almost since their discovery, with microwave diodes ${ }^{12}$ and nanotube field emission displays ${ }^{13}$ reported. Previous work by
Modi et al. ${ }^{14}$ demonstrated detection of a range of gases, including helium, using a gas discharge sensor based on a nanotube array. However, these devices relied on an avalanche cascade mechanism and thus operated in a high-pressure regime that is inaccessible to helium microscopy. Riley et al. ${ }^{15}$ have previously reported the field ionization of helium using carbon nanotubes grown on the end of a wire; resulting in extra field enhancement due to the substrate geometry. Planar nanotube ionization of rubidium (ionization energy $4.18 \mathrm{eV})^{16}$ has been previously demonstrated by the pioneering work of Gruner et al., ${ }^{17}$ however helium, with an ionization energy of $24.6 \mathrm{eV},{ }^{16}$ is much more difficult to ionize due to the exponential dependence of tunneling probability on ionization energy.

Here we demonstrate that a planar array or forest of vertically aligned nanotubes can also generate fields sufficient to ionize helium. The implementation of a planar geometry ionization element is a key step toward the development of a spatially resolved high-sensitivity neutral atom detector, and hence the realization of neutral atom microscopy.

Experimental setup. Our experiments were carried out in an ultrahigh-vacuum chamber with base pressure $2 \times 10^{-10}$ Torr. Helium (99.999\% purity, BOC gases) was leaked into the chamber using a precision leak valve to a level established using an ionization gauge and a helium sensitivity factor of 5.6. The pressure was monitored simultaneously using a residual gas analyzer. The nanotube detector [Fig. 1(a)] consisted of a nanotube forest grown on a $10 \times 10 \times 0.5 \mathrm{~mm}$ $\mathrm{Si}$ wafer using plasma-enhanced chemical vapor deposition (PECVD) sandwiched between a metal sample holder and a sapphire plate spacer with a $8 \times 8 \mathrm{~mm}$ opening in the center. Across the opening was clamped a fine $\mathrm{Ni}$ grid of transmissivity approximately $85 \%$. The spacing $d$ between the nanotube surface and the grid was $0.4 \mathrm{~mm}$. A channel electron multiplier (CEM, Amptek MD-502) was positioned directly above the opening at a distance of approximately $10 \mathrm{~mm}$, mounted on a three-axis manipulator. The $3 \mathrm{~mm}$ diameter entrance of the CEM was grounded and connected to the $\mathrm{Ni}$ grid. For field ionization, a high-positive dc bias was applied to the sample holder using a Spellman $20 \mathrm{kV}$ SL10 power supply. For field emission, currents were collected on the CEM 
(a)
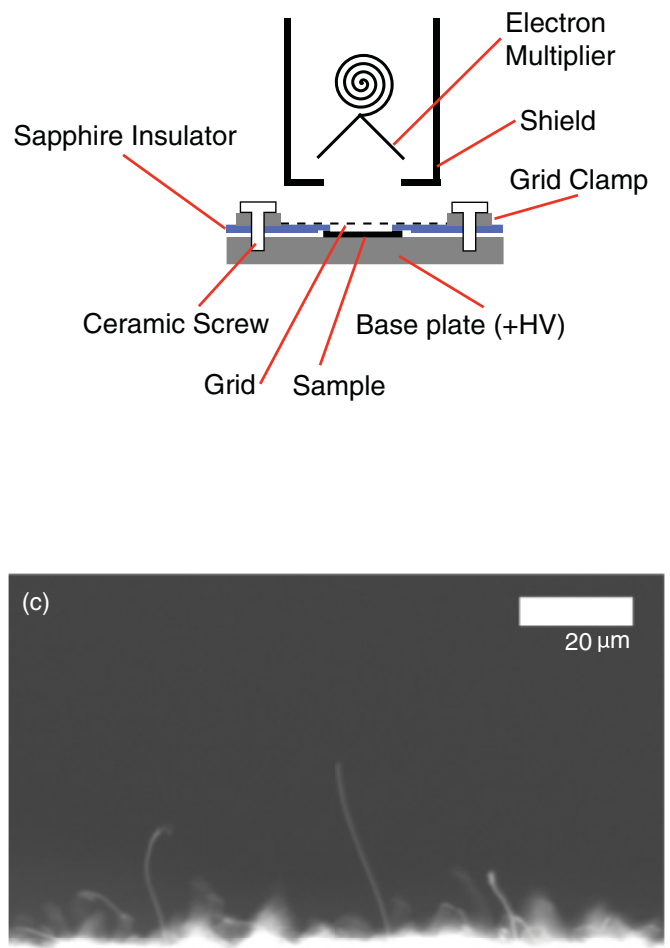
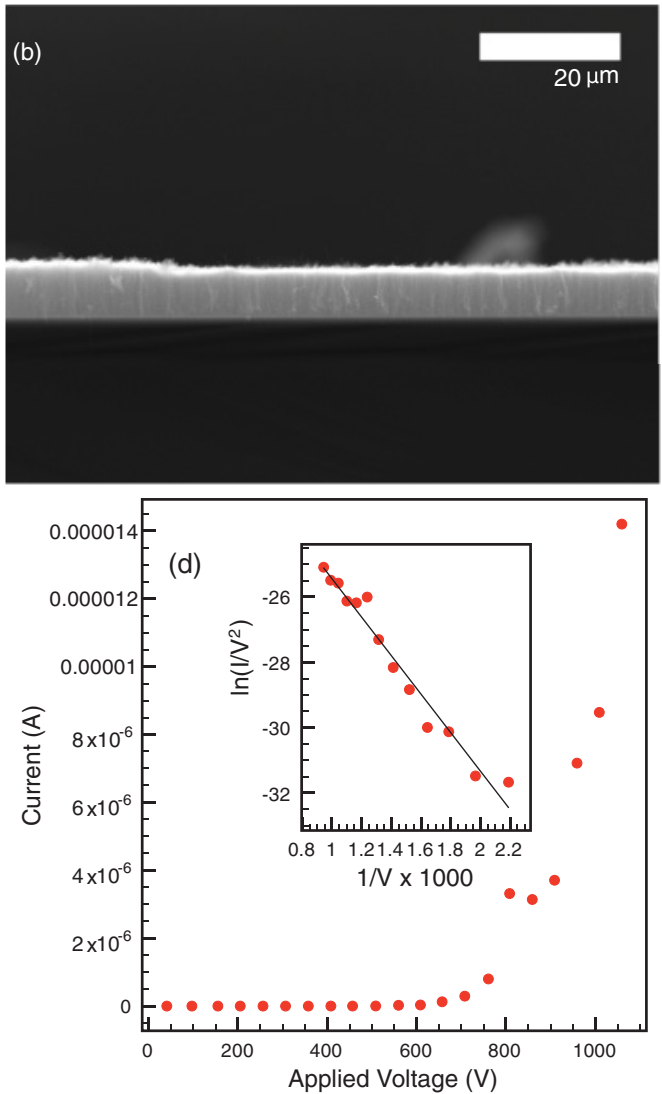

FIG. 1. (Color online) (a) Detector arrangement. The silicon substrate bearing the vertically aligned nanotubes is clamped by a sapphire insulator to the stainless steel base. (b) Nanotube film cross section showing a thick mattress of vertically aligned nanotubes approximately $10 \mu \mathrm{m}$ in length. (c) Magnified image of the film showing that a large number of nanotubes protrude several microns above the bulk nanotube surface. (d) Field emission current-voltage curve from a nanotube sample. The inset shows the Fowler-Nordheim equation fit to the experimental data.

shield [Fig. 1(a)]. The shield was isolated and had a separate electrical connection, thus allowing direct measurement of the impinging field emission current by moving the detector assembly sideways so the lip of the shield was directly over the nanotube forest. Currents were measured using a Keithley 6485 picoammeter. When using the CEM the dark count consisted of random single-count pulses with a mean frequency of approximately $0.1-1.0 \mathrm{~Hz}$.

Silicon substrates with 1-3 $\mathrm{nm}$ of Fe deposited using e-beam evaporation were first cleaned in situ using a argon plasma. Heating to $600{ }^{\circ} \mathrm{C}$ was carried out under $100 \mathrm{sccm}$ of flowing hydrogen with the plasma ignited. Growth lasted for $10 \mathrm{~min}$ using $80 \mathrm{sccm}$ of hydrogen and $20 \mathrm{sccm}$ of ethylene at a plasma power of $180 \mathrm{~W}$. Following $30 \mathrm{~min}$ of hydrogen flow the substrates were annealed at $900{ }^{\circ} \mathrm{C}$ for $30 \mathrm{~min}$ and then cooled to room temperature under $300 \mathrm{sccm}$ of flowing argon. Scanning electron micrographs of the samples show very dense, well-aligned forests of nanotubes approximately $7.5 \mu \mathrm{m}$ in length uniformly coating the surface [Fig. 1(b)]. Particularly long nanotubes were seen at random locations in the forest, sometimes protruding twice as far as the average forest thickness [Fig. 1(c)]. Transmission electron microscopy of the nanotubes revealed they were multiwalled with an average diameter of approximately $20 \mathrm{~nm}$.
Results and discussion. Prior to field ionization, samples were conditioned using extended, high-current field emission (50 $\mu \mathrm{A}$ for $17 \mathrm{~h})$. Current-voltage curves for field emission were collected in order to calculate the field enhancement factor via Fowler-Nordheim analysis. The average of the field emission curves appears in Fig. 1(d). The elementary FowlerNordheim equation gives the field emission current as

$$
I=A a \frac{F^{2}}{\phi} \exp \left(-b \frac{\phi^{3 / 2}}{F}\right),
$$

where $A$ is an approximate emission area, $\phi$ is the emitter workfunction, and $F$ is the local electric field at the emitter surface. Given the sensitivity to $F$, we assume that only the sharpest, most protruding nanotubes contribute significantly to emission. The macroscopic applied field $V / d$ is enhanced by the sharp tips of the nanotubes by a factor $\gamma$, such that

$$
F=\gamma \frac{V}{d}
$$

Substituting Eq. (2) into Eq. (1) we can extract the enhancement factor from the current-voltage data via conventional Fowler-Nordheim analysis. ${ }^{18}$ Assuming $\phi=4.8 \mathrm{eV}$ for the nanotube emitters ${ }^{19}$ and using the Fowler-Nordheim 
constants $^{18} \quad a=1.54 \times 10^{-6} \mathrm{~A} \mathrm{eV} \mathrm{V}^{-2}$ and $b=6.83 \times$ $10^{9} \mathrm{eV}^{-3 / 2} \mathrm{~V} \mathrm{~m}^{-1}$ we obtain a field enhancement factor $(\gamma)$ of approximately 4800.

Such a value is large compared to the expected geometric enhancement from the observed protruding nanotubes, which should be $\approx 300-600$ based on their aspect ratio ${ }^{20}$ and an estimated protrusion length of $7.5 \mu \mathrm{m}$. However, Gruner et al. ${ }^{17}$ measured $\gamma \approx 3900$ for a similar array of nanotubes, and Shearer ${ }^{21}$ measured $\gamma=5800$ for bundles of single-walled nanotubes chemically attached to a silicon substrate, despite none protruding more than a few hundred nanometers from the substrate. Here $A \approx 770 \mathrm{~nm}^{2}$, which indicates that only a handful of nanotubes are involved in field emission and is consistent with our SEM images of the nanotube mat surface. The most likely explanation for the large observed enhancement factors is that only small numbers of nanotubes protrude from the surface and are possibly drawn into alignment under the influence of the applied field.

For a field enhancement factor of 4800 and an electrode spacing of $0.4 \mathrm{~mm}$, the field strength at the tips of the sharpest nanotubes is $1.2 \times 10^{10} \mathrm{~V} / \mathrm{m}$ at $1000 \mathrm{~V}$ applied. Such fields are of the order required to observe field ionization. We found in practice that we could apply up to $3200 \mathrm{~V}$ without generating arcs between the nanotubes and the grid. Thus we can estimate we were generating local fields of up to approximately $3.8 \times 10^{10} \mathrm{~V} / \mathrm{m}$ at the tip of the sharpest nanotubes. This field strength is a factor of approximately 4.3 higher than that estimated by Gruner $e$ al. with the difference most likely due to a smaller electrode spacing $(0.4 \mathrm{~mm}$ vs $1.25 \mathrm{~mm})$ and the narrower diameters of our nanotubes $(20 \mathrm{~nm}$ vs $58 \mathrm{~nm})$.

Figure 2(a) shows a conventional current-voltage curve collected using a tungsten ionizer, at a helium partial pressure of $3.0 \times 10^{-5}$ Torr and a residual background (mostly trace water and hydrogen) pressure of $3.3 \times 10^{-10}$ Torr. The curve in Fig. 2(a) shows the classic two-region shape $\mathrm{e}^{8,9,22,23}$ expected for field ionization consisting of a rapid onset in the field-limited regime where the tunneling rate increases exponentially followed by a more gradual increase in the region limited by the gas supply. ${ }^{24,25}$ The background count rate for the tungsten ionizer in the supply-limited region, from approximately $2400 \mathrm{~V}$ onwards, is of the order of a few counts per second. The peak in the background spectrum at lower voltages corresponds to the field-induced desorption of adsorbates. Figure 2(b) shows a current-voltage curve collected using our nanotube detector arrangement at a background pressure of $1.2 \times 10^{-9}$ Torr, with the curve clearly exhibiting the two-region shape characteristic of field ionization. Moreover, whereas the background count rate for the tungsten ionizer is of the order of a few counts per second, the corresponding count rate for the nanotube array is of the order of 40000 counts/s. Even accounting for the slight difference in background pressure, this increased count rate represents an increased sensitivity to the background gas of over three orders of magnitude for the nanotube array over the tungsten ionizer.

Figure 3 shows the variation of ion current over time compared to the helium partial pressure as measured by the residual gas analyzer. To highlight the linearity of the detector response, we have normalized the ion current by the detector sensitivity factor $\alpha$, where

$$
I=\alpha P_{\mathrm{He}},
$$

and $I$ and $P_{\mathrm{He}}$ are the ion current and partial pressure of helium, respectively. In our case, the sensitivity factor of best fit is $6.2 \times 10^{7}$ counts/Torr. Consistent with previous reports, ${ }^{15,26}$ we find that $\alpha$ is a function of the history of the device and the channel electron multiplier and thus the absolute count rates of Figs. 2(b) and 3 are not directly comparable. The decrease in $\alpha$ is partially due to the degradation of the nanotube emitters and partially due to the degradation of the electron multiplier itself. A solution to the latter was recently published by our group. ${ }^{26}$ Most important is that Fig. 3 shows that the field
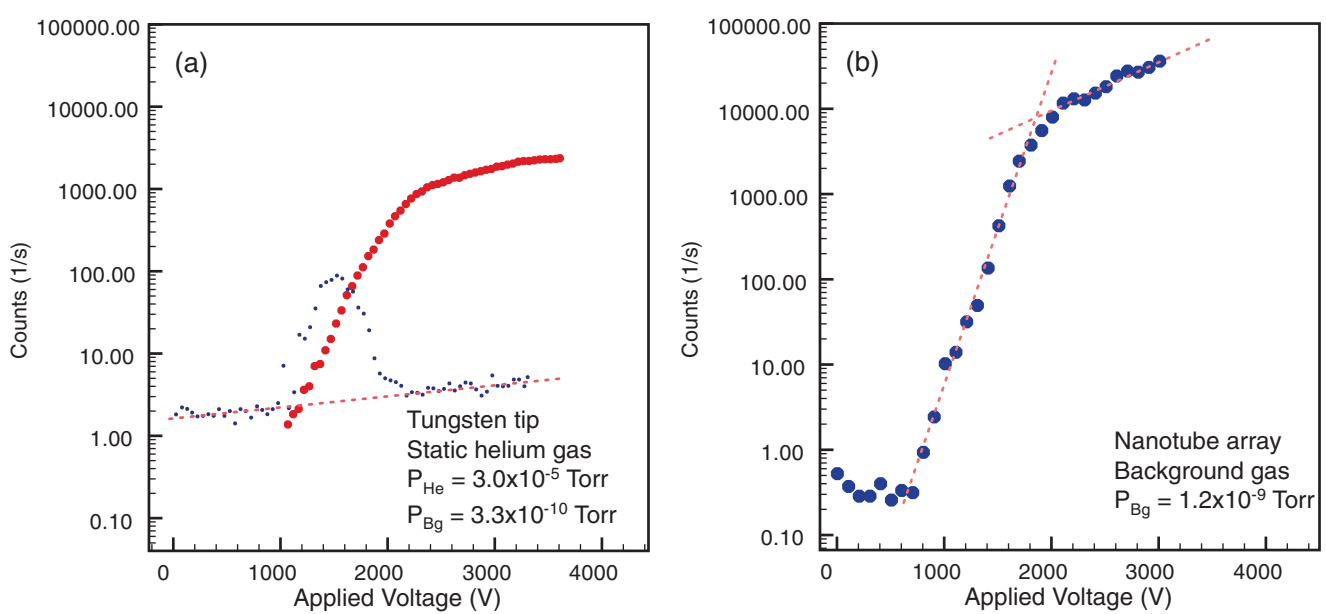

FIG. 2. (Color online) (a) Variation of field ionization current for helium (red dots, pressure $3.0 \times 10^{-5}$ Torr) and background gas (blue dots, pressure $3.3 \times 10^{-10}$ Torr) as a function of applied voltage using a tungsten ionizer. (b) Variation of background field ionization current as a function of applied voltage using the nanotube detector at a background pressure of $1.2 \times 10^{-9}$ Torr. Dashed lines indicate the two characteristic regions of field ionization. The desorption peak in the background of (a) is exaggerated by the logarithmic vertical scale. 


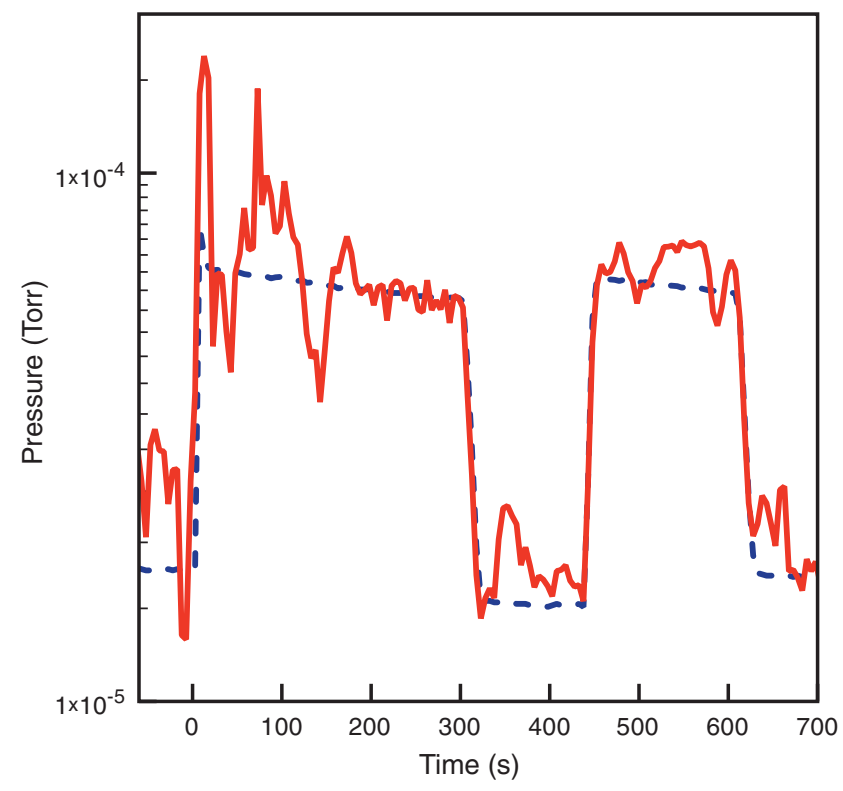

FIG. 3. (Color online) Variation of ion current (red line) compared to the helium partial pressure as measured by a residual gas analyzer (blue dashed line) for a nanotube detector held at an applied voltage of $3200 \mathrm{~V}$. The ion count rate has been normalized using a detector sensitivity factor of $6.2 \times 10^{7}$ counts/Torr.

ionization current signal is clearly correlated with the helium partial pressure.

In addition, while $\alpha$ is essentially constant over time scales of the order minutes, several rapid variations do occur over shorter time scales that are not correlated with pressure changes. These rapid fluctuations in ionization current are also consistent with previous observations ${ }^{15}$ and suggest that the nanotubes involved in ionization undergo rapid changes in the high-field environment. Recent studies of the field ionization of rubidium, a species with a much lower ionization energy than helium, suggest that these rapid variations in ionization current occur even at relatively low applied fields ${ }^{17}$ and may indicate a different mechanism rather than simple nanotube degradation. As such, it is likely that for a practical detector, the nanotube growth will need to be very carefully controlled to produce nanotubes of high quality.

A typical tungsten field ionizer under the same physical conditions as presented here and of a similar end radius $(\approx 10 \mathrm{~nm})$ as the nanotubes generates a helium field ionization current of between 100 and $1000 \mathrm{~Hz}$ [Fig. 2(a)]. Therefore, based on the ionization currents $(\approx 5000 \mathrm{~Hz})$ seen from our detector, we estimate that between 5 and 50 nanotubes were active in ionization. These figures are consistent with the calculated emission area from field emission and are a small fraction of the nanotubes expected to extend from the surface seen in Fig. 1(c), indicating that many orders of magnitude higher current can be expected for devices utilizing well-spaced nanotubes rather than simple forests as prepared here. Nevertheless, in terms of sensitivity our planar detector is already comparable to previously reported work for helium detection using nanotubes grown on the end of a blunt wire. ${ }^{15}$

Conclusions. We have demonstrated field ionization of helium using carbon nanotubes grown on a planar surface. The present device shows the expected response to changes in helium pressure and further refinement of the nanotube growth process should lead to a much more stable ionization detector. The ability to create a planar ionization detector for helium allows for much higher helium detection sensitivity and is an important step in the development of advanced atomic beam techniques including helium microscopy.

Acknowledgments. This research was supported under Australian Research Councils Discovery Projects funding scheme (Project No. DP0881308). Postgraduate research scholarships (KOD, AF, and MB) from the University of Newcastle are also gratefully acknowledged. *paul.dastoor@newcastle.edu.au

${ }^{1}$ D. A. MacLaren, B. Holst, D. J. Riley, and W. Allison, Surf. Rev. Lett. 10, 249 (2003).

${ }^{2}$ J. Braun, P. Day, J. Toennies, G. Witte, and E. Neher, Rev. Sci. Instrum. 68, 3001 (1997).

${ }^{3}$ D. DePonte, S. Kevan, and F. S. Patton, Rev. Sci. Instrum. 77, 055107 (2006).

${ }^{4}$ B. Holst and W. Allison, Nature (London) 390, 244 (1997).

${ }^{5}$ D. Barredo, F. Calleja, P. Nieto, J. Hinarejos, G. Laurent, A. L. V. de Parga, D. Farias, and R. Miranda, Adv. Mater. 20, 3492 (2008).

${ }^{6}$ M. Koch, S. Rehbein, G. Schmahl, T. Reisinger, G. Bracco, W. E. Ernst, and B. Holst, J. Microscopy 229, 1 (2008).

${ }^{7}$ P. Smith, Phys. Rev. 36, 1293 (1930).

${ }^{8}$ M. K. Miller, Atom Probe Field Ion Microscopy (Clarendon, New York, 1996).

${ }^{9} \mathrm{~T}$. Tsong, Atom-Probe Field Ion Microscopy (Cambridge University Press, Cambridge, 1990).

${ }^{10}$ K. Teo, M. Chhowalla, G. Amaratunga, W. Milne, D. Hasko, G. Pirio, P. Legagneux, F. Wyczisk, and D. Pribat, Appl. Phys. Lett. 79, 1534 (2001).
${ }^{11}$ A. Maiti, C. J. Brabec, C. M. Roland, and J. Bernholc, Phys. Rev. Lett. 73, 2468 (1994).

${ }^{12}$ K. Teo, E. Minoux, L. Hudanski, F. Peauger, J. Schnell, L. Gangloff, P. Legagneux, D. Dieumegard, G. Amaratunga, and W. Milne, Nature (London) 437, 968 (2005).

${ }^{13}$ W. Choi, D. Chung, J. Kang, H. Kim, Y. Jin, I. T. Han, Y. H. Lee, J. E. Jung, N. S. Lee, G. S. Park, and J. M. Kim, Appl. Phys. Lett. 75, 3129 (1999).

${ }^{14}$ A. Modi, N. Koratkar, E. Lass, B. Wei, and P. Ajayan, Nature (London) 424, 171 (2003).

${ }^{15}$ D. Riley, M. Mann, D. A. MacLaren, P. C. Dastoor, W. Allison, K. Teo, G. Amaratunga, and W. Milne, Nano Lett. 3, 1455 (2003).

${ }^{16}$ N. I. of Standards and Technology, Physical Reference Data: Elemental Data Index [http://physics.nist.gov/cgi-bin/Elements/ elInfo.pl?element=2\&context=noframes].

${ }^{17}$ B. Grüner, M. Jag, A. Stibor, G. Visanescu, M. Häffner, D. Kern, A. Günther, and J. Fortágh, Phys. Rev. A 80, 063422 (2009).

${ }^{18}$ R. G. Forbes, J. Vac. Sci. Technol. B 17, 526 (1999).

${ }^{19}$ P. Liu, Q. Sun, F. Zhu, K. Liu, K. Jiang, L. Liu, Q. Li, and S. Fan, Nano Lett. 8, 647 (2008). 
${ }^{20}$ F. Read and N. Bowring, Nucl. Instrum. Methods Phys. Res. Sec. A 519, 305 (2004).

${ }^{21}$ C. Shearer, J. Yu, K. O’Donnell, L. Thomsen, P. Dastoor, J. Quinton, and J. Shapter, J. Mater. Chem. 18, 5753 (2008).

${ }^{22}$ E. W. Muller and K. Bahadur, Phys. Rev. 102, 624 (1956).

${ }^{23}$ K. Jousten, K. Bohringer, and S. Kalbitzer, Appl. Phys. B 46, 313 (1988).
${ }^{24}$ H. V. Eekelen, Surf. Sci. 21, 21 (1970).

${ }^{25}$ K. O'Donnell, A. Fahy, L. Thomsen, D. J. O'Connor, and P. C. Dastoor, Meas. Sci. Technol. 22, 015901 (2011).

${ }^{26}$ A. Fahy, K. M. O’Donnell, M. Barr, X. J. Zhou, W. Allison, and P. C. Dastoor, Meas. Sci. Technol. 22, 115902 (2011). 